\title{
Emprego dos cimentos resinosos autoadesivos: uma abordagem sobre a eficácia e os protocolos empregados
}

Employment of autoadesive resin cements: an approach on effectiveness and protocols employed Empleo de los cementos resinosos autoadhesivos: un enfoque sobre la eficacia

\author{
Eloiza Leonardo de MELO' \\ Lídia Dinoah AGUIAR ${ }^{2}$ \\ Mayara Aragão de Lira GOMES ${ }^{3}$ \\ Daiany Catão FURTADO ${ }^{4}$ \\ Kaíse Tavares PONTES ${ }^{3}$ \\ Olimpia Crispim da SILVEIRA ${ }^{5}$ \\ José Lacerda das NEVES ${ }^{3}$ \\ Rodivan BRAZ ${ }^{6}$
}

${ }^{I}$ Mestranda em Odontologia, Área de concentração em Dentística/Endodontia, Universidade de Pernambuco-UPE, 54753-220 Camaragibe-PE, Brasil

${ }^{2}$ Graduanda em Odontologia, Universidade de Pernambuco-UPE, 54753-220 Camaragibe-PE, Brasil

${ }^{3}$ Especialista em Dentística, Instituto de Odontologia da Paraíba-IOP, 58400-126 Campina Grande-PB, Brasil

${ }^{4}$ Mestre em Ciência e Tecnologia em Saúde, Universidade Estadual da Paraíba-UEPB, 58429-500, Campina Grande-PB, Brasil

${ }^{5}$ Professora de Dentística da Universidade Estadual da Paraíba-UEPB, 58429-500, Campina Grande-PB, Brasil

${ }^{6}$ Professor de Dentística da Universidade de Pernambuco, 54753-220, Camaragibe-PE, Brasil

\begin{abstract}
Resumo
Com o avanço da Odontologia Adesiva, através da criação dos cimentos resinosos autoadesivos, ocorreu uma melhoria na interação química com os substratos e os procedimentos que necessitam de um material cimentante, os quais passaram a apresentar sensível melhora, tanto na técnica quanto na garantia de longevidade do procedimento restaurador/reabilitador. Entretanto, assim como os cimentos resinosos convencionais, os autoadesivos apresentam limitações, sendo, portanto, imprescindível o conhecimento das recomendações da literatura atual para uma correta utilização do material sem prejudicar seu desempenho. Este trabalho objetivou através de uma revisão de literatura coletar as informações mais atuais sobre os cimentos resinosos autoadesivos bem como elucidar possíveis limitações destes materiais. Para o esmalte as recomendações encontradas são de realização de condicionamento ácido seletivo. Para a dentina, como se trata de um substrato altamente orgânico, onde o condicionamento ácido pode remover a smear layer e promover uma sensibilidade pós- operatória a aplicação do cimento apenas, parece ser a melhor indicação. Para substratos a base de ligas metálicas e a zircônia a criação de micro retenções ou a utilização de agentes de união química ainda é recomendável devido à fraca interação dos ácidos dos cimentos resinosos autoadesivos com estas superfícies.
\end{abstract}

Descritores: Cimentos Dentários; Cimentos de Resina; Revisão.

\section{Abstract}

With the advancement of Adhesive Dentistry, through the creation of self-adhesive resin cements, there was an improvement in the chemical interaction with the substrates and procedures that require a cementing material, which started to show a significant improvement, both in technique and in the guarantee of longevity restorative/rehabilitation procedure. However, like conventional resin cements, the self-adhesives have limitations and it is therefore imperative to know the recommendations of the current literature for a correct use of the material without impairing its performance. This work aimed at collecting the most current information on self - adhesive resin cements as well as elucidating possible limitations of these materials through a literature review. For the enamel the recommendations found are of performing selective acid conditioning. For dentin, because it is a highly organic substrate, where acidic conditioning can remove the smear layer and promote a postoperative sensitivity to cement application only, it seems to be the best indication. For substrates based on metal alloys and zirconia the creation of micro retentions or the use of chemical bonding agents is still recommended due to the poor interaction of the acids of the selfadhesive resin cements with these surfaces.

Descriptors: Dental Cements; Resin Cements; Review.

\section{Resumen}

Con el avance de la Odontología Adhesiva a través de la creación de los cementos resinosos autoadhesivos ocurrió una mejora en la interacción química con los sustratos y los procedimientos que necesitan un material cementante presentaron sensible mejoría tanto en la técnica y en la garantía de longevidad del procedimiento restaurador/rehabilitador. Sin embargo, así como los cementos resinosos convencionales, los autoadhesivos presentan limitaciones, siendo, por lo tanto, imprescindible el conocimiento de las recomendaciones de la literatura actual para una correcta utilización del material sin perjudicar su desempeño. Este trabajo objetivó a través de una revisión de literatura recoger las informaciones más actuales sobre los cementos resinosos autoadhesivos así como elucidar posibles limitaciones de estos materiales. Para el esmalte las recomendaciones encontradas son de realización de condicionamiento ácido selectivo. Para la dentina, como se trata de un sustrato altamente orgánico, donde el condicionamiento ácido puede quitar la smear layer y promover una sensibilidad postoperatoria la aplicación del cemento, apenas, parece ser la mejor indicación. Para sustratos a base de aleaciones metálicas y zirconia la creación de micro retenciones o la utilización de agentes de unión química todavía es recomendable debido a la débil interacción de los ácidos de los cementos resinosos autoadhesivos con estas superficies.

Descriptores: Cementos Dentales; Cementos de Resina; Revisión.

\section{INTRODUÇÃO}

As restaurações indiretas com alto desempenho estético vêm ganhando cenário na odontologia há cerca de duas décadas, corroborando para a necessidade de utilização de um cimento resinoso que garanta longevidade e sucesso clínico a longo prazo. Os cimentos autoadesivos, por sua vez, devido às propriedades de resistência mecânica ao conjunto dente/restauração, redução na complexidade da técnica de cimentação e adesão aos múltiplos substratos em situações desfavoráveis, como ausência de um bom isolamento, preparos muito subgengivais e presença de fluidos são os mais utilizados atualmente, pela proposta de passo único e redução da sensibilidade da técnica ${ }^{1,2}$.

Componentes essenciais como tipo e tamanho de partículas de carga, tipos de metacrilatos 
e, principalmente, a presença de monômeros ácidos como o 10-MDP (10-Metacriloxiedecil Dihidrogenio Fosfato), 4-META (4-Metacriloxietil) são responsáveis pela adesão micromecânica entre o cimento e o substrato. E estes últimos (monômeros ácidos) promovem um padrão de desmineralização considerado aceitável e cria uma união química com o cálcio da hridoxiapatita, formando precipitados resistentes e insolúveis em água ${ }^{3}$.

Os cimentos autoadesivos nos estágios iniciais são extremamente ácidos, apresentam um baixo PH e alta hidrofilia. Entretanto, com a evolução da reação, o PH sofre elevação, os monômeros ácidos começam a ser degradados pelas reações químicas e o material passa a adquirir características hidrofóbicas, reduzindo a sorção em água, a expansão higroscópica e a degradação hidrolítica ${ }^{1}$.

Tendo em vista o aumento na utilização destes materiais e a pouca informação sobre o mecanismo de adesão dos cimentos com os diferentes substratos, o presente estudo se propôs a realizar uma revisão de literatura sobre a avaliação da eficácia dos cimentos resinosos autoadesivos nos diferentes substratos envolvidos na cimentação de restaurações indiretas, bem como o protocolo de cimentação utilizado para cada substrato.

\section{REVISÃO DA LITERATURA}

Os cimentos resinosos são materiais relativamente novos, que foram introduzidos no mercado com a proposta de simplificação e unificação do procedimento de cimentação de restaurações indiretas a base de resina, zircônia, metais e pinos intraradiculares. A adição de monômeros ácidos a composição destes materiais eliminou a etapa de condicionamento ácido separadamente, melhorando a manipulação do material, mantendo propriedades mecânicas e estéticas semelhantes aos cimentos resinosos convencionais e sem qualquer sensibilidade pósoperatória relatada ${ }^{4,5}$.

A adição de monômeros ácidos como o 10MDP, 4-META, BMP, PENTA P, PHENYL-P e PMGDM capazes de promover uma desmineralização na dentina sem causar sensibilidade pós-operatória são equilibrados de modo a não promover hidrofilia excessiva a ponto de comprometer os valores de adesão e manter padrões aceitáveis de desmineralização ${ }^{5,6}$. Entretanto, outros fatores como tipos de precipitação da substância e limitação na descalcificação dos substratos trazem preocupação em relação ao comportamento destas reações sobre dentes vitais como também sobre a redução dos valores de adesão pela difusão limitada do cimento na presença de smear layer ${ }^{7,8}$.

Portanto os cimentos autoadesivos nos estágios iniciais são extremamente ácidos, apresentam um baixo $\mathrm{PH}$ e alta hidrofilia. Com a evolução da reação, o PH sofre elevação, os monômeros ácidos começam a ser degradados pelas reações químicas e o material passa adquirir características hidrofóbicas, reduzindo a sorção em água, a expansão higroscópica e a degradação hidrolítica ${ }^{1}$.

O mecanismo de polimerização dos cimentos traz discussões devido à "competição" entre os dois mecanismos de polimerização. Quando o material é fotoativado são formadas redes poliméricas onde os componentes responsáveis pela polimerização química ficam aprisionados, perdendo a mobilidade e retardando a completa polimerização, chegando a um tempo aproximado de reação de 24 horas até 7 dias. $\mathrm{O}$ aprisionamento dos ativadores químicos, bem como dos monômeros ácidos residuais gera preocupações porque não se sabe a influencia destes remanescentes em uma possível hidrólise ou toxicidade quando se trata de dentes vitais ${ }^{1,2,6}$.

O esmalte dentário, tecido constituído predominantemente por hidroxiapatita (forma cristalizada do fosfato de cálcio) é um substrato cujo condicionamento com ácido fosfórico $37 \%$ já é consagrado e bem aceito como protocolo efetivo para sua desmineralização ${ }^{3,6}$. Entretanto, os monômeros ácidos incluídos nos cimentos autoadesivos não são capazes de promover padrões de desmineralização equivalentes aos obtidos com o condicionamento com ácido fosfórico, interagindo apenas com as regiões mais superficiais dos tecidos duros ${ }^{1,6}$.

Embora Souza et al. ${ }^{9}$ tenham concluído em seu estudo que a desproteinização da dentina não produz efeitos negativos nos cimentos avaliados em seu estudo, pode não ser uma boa opção para dentes vitais, devido ao risco de promover uma sensibilidade pós-operatória no paciente.

Além disto, o mecanismo de hibridização dos cimentos autoadesivos, quando comparados aos métodos separados de condicionamento ácido são considerados semelhantes aos padrões obtidos nos adesivos autocondicionantes, observando-se baixa hibridização interprismática e baixa resistência adesiva. Portanto, o condicionamento seletivo com ácido fosfórico $37 \%$ é recomendado quando os cimentos autoadesivos são utilizados ${ }^{1,2,6}$.

Já na dentina o condicionamento ácido separado promove a criação de matrizes colágenas que reduzem o potencial de penetração dos materiais viscosos e a resistência adesiva destes, promovendo uma interface fraca ${ }^{2,6}$. A utilização de ácido poliacrílico parece ter um impacto melhor nos valores de resistência adesiva, entretanto já que em dentes vitais se preconiza a manutenção da smear layer tal procedimento pode removê-la por completo, podendo haver uma possível sensibilidade dentinária ${ }^{1}$.

A ação dos cimentos autoadesivos sobre a dentina promove valores favoráveis de resistência à adesão, embora não produza uma camada híbrida 
consistente $^{6}$ e apresente graus de difusão limitada entre os cimentos comercializados ${ }^{7}$. Os possíveis fatores que impedem esta difusão são o grau de viscosidade do material, a rápida reação ácido-base, remanescente de outros cimentos utilizados anteriormente, o rápido efeito de neutralização pela liberação de água durante o processo, aumento do $\mathrm{PH}$ e da alcalinidade com a evolução da reação ou pela composição da smear layer ${ }^{6,7,10}$.

Existem poucos dados na literatura sobre a adesão destes cimentos com metais, entretanto existe consenso em afirmar que os valores de adesão quando comparados aos dos cimentos de fosfato de zinco, ionômero de vidro e óxido de zinco e eugenol são maiores ${ }^{6}$. Portanto, em casos que metais estejam presentes no processo de cimentação, o jateamento com partículas de óxido de alumínio ou a utilização de primers para metais é recomendado ${ }^{1,7}$.

Para a zircônia as recomendações são do jateamento com partículas abrasivas e a aplicação de primers. Blatz et al. ${ }^{5}$ avaliaram a resistência adesiva de seis cimentos resinosos autoadesivos para zircônia com jateamento de partículas abrasivas e sem o jateamento. Concluíram que a resistência adesiva dos cimentos aumentou com a abrasão de partículas, especificamente quando os cimentos contendo MDP e 4-META foram utilizados.

Ferracane et al. ${ }^{6}$ afirmam que, quando se trata de cerâmica, a maioria dos estudos utiliza a zircônia e a resistência adesiva dependente da marca comercial estudada, sendo o RelyX Unicem que promove melhores valores de adesão, principalmente quando é feito o jateamento com partículas abrasivas ou silicatização. Entretanto não estão esclarecidos os efeitos da deterioração e exposição às diversas condições da cavidade bucal sobre a retenção das restaurações indiretas em longo prazo. Estudos in vitro não são capazes de fornecer esta informação.

Em pinos intraradiculares de fibra de vidro ou de quartzo a adesão química promove valores de resistência de união aceitáveis comprovados por testes de push-out. A resistência adesiva nestes casos é influenciada pelo cimento resinoso autoadesivo pelo espaço a ser ocupado pelo pino e pela interação entre estes dois fatores ${ }^{11}$.

Kahnamouei et al. ${ }^{11}$ avaliaram a resistência adesiva de pinos de fibra de quartzo no canal radicular com cimentos autocondicionantes e autoadesivos. Concluíram que os cimentos resinosos autoadesivos promovem alta resistência de união ao push-out na região apical; já os autoadesivos resultaram em uma resistência de união mais uniforme nas diferentes regiões do canal radicular.

Em cerâmicas ácido-sensíveis o mecanismo de adesão está ligado à ação sobre as partículas de vidro ácido-soluveis associada à união química pelo silano. Zorzinet al. ${ }^{12}$ avaliaram o desempenho adesivo dos cimentos autoadesivos para zircônia e dissilicato de lítio nos modos de polimerização dual e autopolimerizável, antes e depois da termociclagem. Concluíram que para a zircônia a resistência adesiva foi melhor quando utilizados os cimentos duais, o mesmo não acontece para o dissilicato de lítio.

Fatores como utilização de clorexidina $2 \%$ ou agentes quelantes como tratamento de superfície, influencia de materiais cimentantes provisórios, influencia do tempo entre a manipulação, influência do modo de cura na energia de superfície também são fatores que influenciam diretamente no processo de cimentaçã ${ }^{13-15}$.

Youm et al. ${ }^{13}$ avaliaram o efeito do modo de cura e diferentes pré-tratamentos na resistência a microretenção dos cimentos autoadesivos. Concluíram que com o RelyX U200 o pré-tratamento com EDTA ou ácido poliacrílico pode ser recomendada para aumentar a resistência de união do cimento à dentina.

Fonseca et al. $^{14}$ avaliaram o grau de conversão, absorção e solubilidade e água dos cimentos autoadesivos sujeitos a diferentes tempo de intervalo entre a preparação do material e a fotoativação. Concluíram que o tempo entre a manipulação e a fotoativação influenciam significativamente o grau de conversão dos cimentos resinosos. Em geral, recomenda-se a fotoativação o mais breve possível após a manipulação.

Saker et al. ${ }^{15}$ testaram o efeito de diferentes agentes quelantes na resistência a microtração dos cimentos para a dentina. Concluíram que a quitosana, EDTA $17 \%$ ou ácido poliacrílico podem ser utilizados como agentes quelantes quando da cimentação de cerâmicas com cimentos autoaesivos.

Di Hipólito et al. ${ }^{16}$ investigaram a resistência a microtração e o padrão de falha dos cimentos autoadesivos em dentina pré-tratada com diferentes concentrações de clorexidina. Concluíram que a dentina quando tratada com clorexidina $0,2 \%$ ou 2,0\% apresentou baixa ligação quando associadas aos cimentos resinosos testados.

Takimoto et al. ${ }^{17}$ avaliaram a energia livre de superfície e a resistência adesiva a dentina após a remoção dos cimentos provisórios. Concluíram que a presença de cimento provisório interfere na adesão dos cimentos autoadesivos com a dentina. Deve-se ter cuidado com remanescentes de cimento temporário quando for utilizar os cimentos autoadesivos

Indiscutivelmente, os cimentos autoadesivos possibilitaram a redução no número de passos clínicos durante o procedimento de cimentação de restaurações indiretas. Tal benefício, no entanto, ainda não eliminou a necessidade de tratamento de substratos como a zircônia, o dissilicato de lítio, o esmalte e os metais, como demonstra o estudo de Manso e Carvalho ${ }^{1}$. Este achado indica que tenhamos cautela na utilização dos cimentos resinosos 
autoadesivos principalmente quando tratarmos de substratos desfavoráveis para a cimentação.

Dentre os monômeros ácidos, o mais citado na literatura e que promove melhor adesão química pela interação com o cálcio da hidroxiapatita é o 10 MDP. Tal afirmação pode ser ratificada pelos estudos de Manso e Carvalho ${ }^{1}$, Leeet al. ${ }^{18}$ e Ferracane et al. ${ }^{6}$. Portanto, parece recomendável a escolha de materiais que contenham o 10-MDP em sua composição. Os demais monômeros ácidos necessitam de mais estudos sobre o mecanismo de adesão.

Preocupações permanecem em relação à cimentação de restaurações indiretas em dentes vitais, já que a incorporação da smear layer nestas situações é recomendável para que se previna uma sensibilidade pós-operatória. Propriedades dos materiais, tal como a viscosidade, podem ser fatores limitantes da difusão dos cimentos sobre esta lama dentinária afetando negativamente as propriedades mecânicas e a formação dos tags, fato este também citado nos estudos de Vieira-Filho et $\mathrm{al}^{2}$. Assim, parece ser recomendável a utilização de ácido poliacrílico em dentina, mas em casos de dente desvitalizado.

Como mostra Manso e Carvalho ${ }^{1}$, a aplicação de primers para zircônia, metal e a utilização de agentes de união como o silano ainda são recomendáveis quando for utilizar os cimentos resinosos autoadesivos. Também ainda é recomendável a realização de condicionamento seletivo em esmalte, já que os monômeros ácidos tem baixo potencial de desmineralização sobre o esmalte, corroborando com os achados deste estudo. A adesão a estes substratos permanece um desafio.

Fatores como o tempo entre a manipulação do material e a fotopolimerização exercem influência importante no endurecimento precoce, sendo, portanto recomendável rápida manipulação $\mathrm{e}$ fotoativação o mais breve possível para evitar a polimerização precoce, como comprovado por Fonseca et al., ${ }^{14}$.Sabendo-se que se trata de uma propriedade inerente aos materiais resinosos de cura dupla, é importante estar atento ao tempo de polimerização química do material. Alguns autores recomendam também que seja necessária a aplicação de uma força compressiva sob a restauração indireta para que o material possa penetrar melhor nos túbulos dentinários e assim melhorar o embrincamento mecânico. Entretanto, na maioria dos artigos essa força compressiva não está bem definida, já que alguns autores recomendam "leve pressão" ou "pressão digital", mas ainda não se sabe exatamente a quantidade de força necessária durante 0 assentamento da peça ${ }^{19,20}$.

É fato que devemos levar em consideração diversos fatores envolvidos no momento da cimentação, já que é um passo sensível e que exige um protocolo rigoroso. Contudo a utilização dos cimentos resinosos autoadesivos ainda apresenta melhores resultados de resistência adesiva quando comparado a outros materiais utilizados durante anos nos procedimentos de cimentação ${ }^{6,18}$.

\section{CONSIDERAÇÕES FINAIS}

Os cimentos resinosos autoadesivos, embora tenham trazido facilidade no procedimento de cimentação ainda deve ser realizado com cautela respeitando as recomendações de tratamento de superfície dos diversos substratos.A utilização de materiais que contenham 10 MDP é recomendada para melhorar a adesão química aos substratos. Os demais monômeros ácidos ainda permanecem com pouca informação na literatura.

Em esmalte permanece a recomendação de condicionamento seletivo e na dentina, quando se trata de dentes vitais não é recomendável qualquer tipo de tratamento de superfície, exceto em casos de dentes tratados endodonticamente, a utilização de ácido poliacrílico é indicada para melhorar a resistência de união

\section{REFERÊNCIAS}

1. Manso AP, Carvalho RM. Dental cements for luting and bonding restorations self-adhesive resin cement. Dent Clin North Am. 2017; 61(4):821-34.

2. Vieria Filho WS, Alonso RCB, Gonzaléz AHM, D'alpino PHP, Di Hipólito V. Bond strength and chemical interaction of self-adhesive resin cements according to the dentin region. Int $\mathrm{J}$ Adhes Adhes. 2017; 73:22-7.

3. Zayetev D, Panfilov P. Anisotropy of the mechanical properties of human dental enamel. Mater Lett. 2015; 159:428-31.

4. Hattar S, Hatamleh M, Khraisat A, Al-Rabah M. Shear bond strength of self-adhesive resin cements to base metal alloy. J Prosthet Dent. 2014; 111(5):411-15.

5. Blatz MB, Mante FK, Saleh N, Atlas AM, Mannan S, Ozer F. Postoperative tooth sensitivity with a new self-adhesiveresin cement - a randomized clinical trial. Clin Oral Investig. 2013; 17(3):793-98.

6. Ferracane JL, Stansbury JW, Burke FJT. Selfadhesive resin cements - chemistry, propertiesand clinical considerations. J Oral Rehabil. 2011; 38(4):295-314.

7. Monticelli F, Osorio R, Mazzitelli C, Ferrari M, Toledano M. Limited decalcification/diffusion of self-adhesive cements into dentin. J Dent Res. 2008; 87(10):974-79.

8. Mazzitelli C, Monticelli F, Osorio R, Casucci A, Toledano M, Ferrari M. Effect of simulated pulpal pressure on self-adhesive cements bonding to dentin. Dental Mater. 2008; 24(9):1156-63.

9. De Souza FB, Delfino CS, Turbino NL, Braz R. 
Deproteinized dentin: a favorable substrate to self-bonding resin cements? J Biomed Mater Res B Appl Biomater. 2011; 98(2):387-94.

10. Marghalani HY. Sorption and solubility characteristics of self-adhesive resincements. Dental Mater. 2012; 28(10):e187-98.

11. Kahnamouei MA, Mohamadi N, Navimipour EJ, Shakerifar M. Push-out bond strength of quartz fibre posts to root canal dentin using totaletchand self-adhesive resin cements. Med Oral Patol Oral Cir Bucal. 2012; 17(2):e337-44.

12. Zorzin J, Belli R, Wagner A, Petchelt A, Lohbauer U. Self-adhesive resin cements: adhesive performance to indirect restorative ceramics. J Adhes Dent. 2014; 16(6):541-46.

13. Youm SH. Jhung KH, Son SA, Kwon YH, Park JK. Effect of dentin pretreatment and curing mode on the microtensile bond strength of selfadhesive resin cements. J Adv Prosthodont. 2015; 7(4):317-22.

14. Silva Fonseca AS, Mizrahi J, Menezes LR, Valente LL, de Moraes RR, Schneider LF. The effect of time between handling and photoactivation on self-adhesive resin cement properties. J Prosthodont. 2014; 23(4):302-7.

15. Saker S, Alnazawi A, Ozean M. Adhesive strength of self-adhesive resins to lithium disilicateceramic and dentin: effect of dentin chelating agents. Odontology. 2016; 104(1):53-9.

16. Di Hipólito V, Rodrigues FP, Piveta FB, Azevedo LC, Alonso RCB, Silikas N et al. Effectiveness of self-adhesive luting cements in bonding tochlorhexidine-treated dentin, Dental Mater. 2012; 28(5):495-501.

17. Takimoto $M$, Ishii $R$, Iino $M$, Shimizu $Y$, Tsujimoto A, Takamizawa $\mathrm{T}$ et al. Influence of temporary cement contamination on thesurface free energy and dentine bond strength of selfadhesive cements. J Dent. 2012; 40(2):131-38.

18. Lee SE, Bae JH, Choi JW, Jeon YC, Jeong CM, Yoon MJ et al. Comparative shear-bond strength of six dental self-adhesive resin cements to zirconia. Materials (Basel). 2015; 8(6):3306-15.

19. Goracci C, Cury AH, Cantoro A, Papacchini F, Tay FR, Ferrari M. Microtensile bond strength and interfacial properties of self-etching and selfadhesive resin cements used to lute composite onlays under different seating forces. J Adhes Dent. 2006; 8(5):327-35.

20. Campos RE. Adaptação marginal e resistência à fratura de coroas totais executadas em diferentes sistemas [tese]. Arararaquara: Faculdade de Odontologia de Araraquara, UNESP; 2005.

\section{CONFLITO DE INTERESSES}

Os autores declaram não haver conflitos de interesse.

AUTOR PARA CORRESPONDENCIA

Eloiza Leonardo de Melo

eloizaleonardo@gmail.com

Submetido em 04/07/2018

Aceito em 04/10/2018 